5 - ORIGINAL ARTICLE

ISCHEMIA-REPERFUSION

\title{
Animal model of chronic kidney disease using a unilateral technique of renal ischemia and reperfusion in White New Zealand rabbits ${ }^{1}$
}

\author{
Eliane Barbosa Togoe ${ }^{\mathrm{I}}$, Iandara Schettert Silva ${ }^{\mathrm{II}}$, Juliana Loprete Cury ${ }^{\mathrm{III}}$, Albert Schiaveto Souza ${ }^{\mathrm{IV}}$, José Henrique Saraiva \\ Borges $^{\mathrm{v}}$, Klaus Casaro Saturninovi
}

DOI: http://dx.doi.org/10.1590/S0102-8650201400160005

\begin{abstract}
IMaster, Health and Development Postgraduate Program, Federal University of Mato Grosso do Sul (UFMS), Campo Grande-MS, Brazil. Conception and design of the study, acquisition and interpretation of data, manuscript preparation.

${ }^{I} \mathrm{PhD}$, Postgraduate Program in Health and Development, UFMS, Campo Grande-MS, Brazil. Guidance and experimental design of the study, veterinary conductor of the experimental surgery.

II'Master, Fellow PhD degree, Postgraduate Program in Health and Development, UFMS, Campo Grande-MS, Brazil. Associate Professor, Physiotherapy Department, University Center of Grande Dourados (UNIGRAN), Dourados-MS, Brazil. Design of the study, interpretation of data, critical revision. ${ }^{\mathrm{IV}} \mathrm{PhD}$, Associate Professor, Department of Morphophysiology, UFMS, Campo Grande-MS, Brazil. Statistical analysis.

${ }^{\mathrm{v}} \mathrm{PhD}$, Associate Professor, Veterinary Hospital Unit, UNIGRAN, Dourados-MS, Brazil. Veterinary assistance in the experimental study.

${ }^{\mathrm{V}}$ Master, Associate Professor, Department of Veterinary, UNIGRAN, Dourados-MS, Brazil. Histopathological examinations.
\end{abstract}

\section{ABSTRACT}

PURPOSE: To establish a model of chronic kidney disease in White New Zealand rabbits, using an exclusive unilateral technique of renal ischemia and reperfusion.

METHODS: Twenty males White New Zealand rabbits were used. All animals were subjected to the following: pre-surgical blood collection ( $1^{\text {st }}$ collection) for creatinine and urea serum analysis, left renal ischemia and reperfusion surgery technique, another blood sample was collected after 6 weeks post surgery $\left(2^{\text {nd }}\right.$ collection), the last blood sample ( $3^{\text {rd }}$ collection) blood sample was taken 11 weeks post surgery (pre-euthanasia), euthanasia and withdrawal of right and left kidney for histopathological analysis.

RESULTS: The creatinine levels after surgery was statistically significant higher in the $3^{\text {rd }}$ collection, regarding the $1^{\text {st }}$ and $2^{\text {nd }}$ collection $(\mathrm{p}<0.05)$. A significant statistic increase for urea was showed only in the $2^{\text {nd }}$ collection $(\mathrm{p}<0.05)$ when compared to the $1^{\text {st }}$ and $3^{\text {rd }}$ collections. Histopathological analysis showed bilateral lesions in the renal tissue, consistent to the process of ischemia and reperfusion.

CONCLUSION: This exclusive unilateral technique of renal ischemia and reperfusion without nephrectomy in White New Zealand rabbits, showed effectiveness in getting an animal model of chronic kidney disease

Key words: Renal Insufficiency, Chronic. Models, Animal. Rabbits. 


\section{Introduction}

Chronic Kidney Disease (CKD) is considered one of the major health problems in the world and a significant cause of morbidity and mortality. According to the last two Brazilian Dialysis Census, published in 2012 and 2014, the number of people on dialysis in Brazil has progressively increasing over the years, with an incidence rate of 149 patients per million person ${ }^{1,2}$. CKD is a worldwide epidemy that can be explained by the significant growth of the diabetes mellitus prevalence, cardiovascular disease numbers and the increase of worldwide life expectancy ${ }^{3}$.

The course of CKD is characterized by irreversible, progressive and slow loss of the renal function, which affects diverse systems of the organism, becoming a pathology of difficult approach since the CKD patient may present complications in multiple organs and functions ${ }^{4}$. Thus, the $\mathrm{CKD}$ approach requires a wide variety of interventions, which can be difficult to study in human design methodology.

In view of this problem, animal models of CKD by surgical intervention to reproduce its clinical and pathology has been developed, and thus, therapeutic methods tested. There are some protocols in the literature for the development of an animal model of $\mathrm{CKD}$ in rats. A two time surgery in which the animal is first subjected to performing ischemia and reperfusion surgery, and after a period ranging from four to 12 weeks, the animal undergo a new surgical procedure to accomplish reduction of renal mass by nephrectomy at different rates $2 / 3,4 / 5,5 / 6^{5,6}$. This type of surgical technique results in a reduction of animal's survival and in association with a low volemia of rats jeopardizes the use of this animal model on certain types of experiments, especially methodologic designs requiring long periods of observation. Considering these difficulties, regarding the volemia and survival after experimental induction, it has been proposed, the development of animal models of renal failure in other animal species that can respond more adequately to all necessary procedures.

The White New Zealand rabbit is an alternative in animal models of CKD, as it has the best prospect of drafting technique and survival after CKD induction, besides it offers a therapeutic window of 12 weeks and greater blood volemia compared to rats, which favors the consecutive blood samples for biochemical analysis?

Renal ischemia is the major cause of CKD, triggered by the loss of oxygen supply, nutrients and the removal of wasting products from the renal cells. The tubular epithelium plays a main role in the development of renal deterioration after ischemic injury, especially through cytokines production ${ }^{8,9}$. There are a lot of states that may generate physiopathological renal ischemia / reperfusion, among them, Hypertension and Diabetes Mellitus, which are the two leading causes of CKD in humans and increasingly prevalent among Brazilian people ${ }^{10,11}$.

Thus, the aim of this study was to establish a model of CKD in White New Zealand rabbits, using an exclusive unilateral technique of renal ischemia and reperfusion.

\section{Methods}

The study was approved by the Animal Use Committee of Mato Grosso do Sul Federal University (UFMS), under \# 280 protocols. Twenty male White New Zealand rabbits, weighing 2.5 to $3 \mathrm{~kg}$, sourced from Maringa State University (UEM) were used. The animals were kept in individual cages in a room with constant ventilation and appropriate temperature, with free access to water and commercial feed.

All animals were subjected to the following procedures: first pre-surgery blood sample collection, surgery to access the left kidney and ischemia/reperfusion process, second blood collection after 6 weeks post surgery, third blood sample after 11 weeks of post-surgery (pre-euthanasia), euthanasia. The blood samples were used for creatinine and urea serum measurements.

The first blood sample was taken immediately before surgery, after the administration of the following preanesthetic medications: intramuscular injection with acepromazine $(0.05 \mathrm{~g} /$ $\mathrm{kg}$ ) associated with butorphanol $(1 \mathrm{mg} / \mathrm{Kg}), 15$ minutes before anesthesia. Blood samples were obtained by cardiac puncture, performed by the veterinarian in charge of the research. To the blood sample collection, the following materials were used: disposable syringe, needle $25 / 7$, descarpak, anticoagulant tube for blood collection, cotton and alcohol $70 \%$ and procedure gloves.

For anesthesia, the following medications were used: dissociative anesthetic (tiletamine hydrochloride and zolazepam hydrochloride) at a dose of $5 \mathrm{mg} / \mathrm{kg}$ by intramuscular injection. To access the left kidney, a left laparotomy was performed by a longitudinal incision of $5 \mathrm{~cm}$. After location of the renal hilum, clamping of the vascular pedicle on the left kidney was held during twenty minutes to generate renal ischemia. After this period the clamps were removed, reperfusion of the kidney and subsequent plans synthesis with a 4-0 mononylon. The entire surgical procedure was performed by the veterinarian responsible for the research and co-workers in the Veterinary Surgical Center at the Veterinary Hospital of the University of Grande Dourados (UNIGRAN).

The animals were kept under observation for six weeks after surgery, receiving daily hygiene care, free access to water and commercial feed. At the end of this step, the second blood collection was performed the same way of the first one. 
After more five weeks observation, the third and last blood pre-euthanasia collection was taken. The euthanasia was performed by intravenous barbiturate overdose $(100 \mathrm{mg} / \mathrm{kg})$, followed the withdrawal of the right and left kidneys for histopathology.

The first collection represents normal values for the studied sample, the second and third ones represent the variation of these parameters as a function of time after renal and ischemia/ reperfusion surgical procedure in the left kidney.

After the histopathological material collection, the pieces were immersed in $10 \%$ formaldehyde and processed for paraffin blocking $(5 \mathrm{~cm})$. Then the pieces cut on microtome at 5 micrometers thickness and stained with hematoxylin and eosin (HE).

A pathologist evaluated the slides using Nikon Eclipse E200 electron microscope and the blades analysis used to establish the presence or absence of renal lesions in both kidneys.

To summarize the data of quantitative variables of urea and creatinine, the mean value and standard deviation were used. For statistical comparison of the three time-collection of these variables were used the One-way ANOVA for repeated measure with post hoc Tukey test, considering significance statistic set $\alpha=5 \%$ $(\mathrm{p}<0.05)$. Qualitative variables concerning the histopathological analysis were summarized in absolute and relative frequency (\%).

\section{Results}

A total of twenty rabbits underwent the ischemia and reperfusion surgeries, three of them died throughout the research and were excluded from the final analysis.

The animal model of CKD in White New Zealand rabbits showed good survival and allowed consecutive blood collections without compromising the blood volemia of the animal. In Figure 1 , the change in color of the left kidney after 20 minutes of ischemia during the surgical procedure was observed.

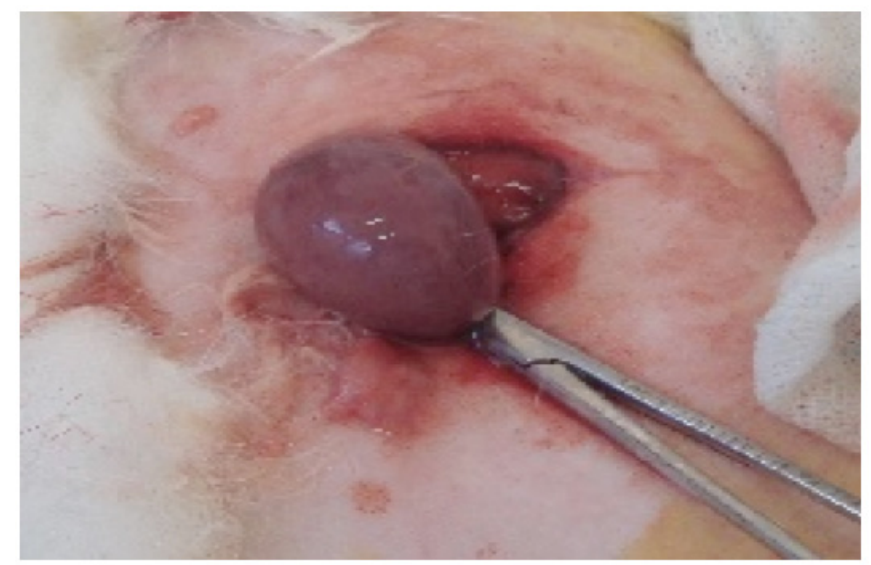

FIGURE 1 - Picture showing the change in coloration of the left kidney after 20 minutes of ischemia and reperfusion procedure.
The results for serum creatinine levels showed a gradual increase after surgery, and the values of the $3^{\text {rd }}$ collection were higher and statistically significant $(\mathrm{p}<0.05)$ compared to the $1^{\text {st }}$ and $2^{\text {nd }}$ collection (Table 1). This value demonstrates a decline in glomerular filtration rate over time.

TABLE 1 - Table showing the mean value and standard deviation of creatinine and urea levels in the three collections.

\begin{tabular}{cccc}
\hline & $1^{\text {st }}$ Collection & $2^{\text {nd }}$ Collection & $3^{\text {rd }}$ Collection \\
\hline Creatinine & $1.01 \pm 0.16$ & $1.15 \pm 0.16$ & $1.73 \pm 0.95^{*}$ \\
Urea & $31.0 \pm 4.53$ & $39.61 \pm 3.63 \#$ & $32.62 \pm 5.79$ \\
\hline
\end{tabular}

Creatinine and urea values presented in $\mathrm{mg} / \mathrm{dl}$

*Creatine values statically significant $(\mathrm{p}<0.05)$ in comparison to the $1^{\text {st }}$ and $2^{\text {nd }}$ collection \#Urea values statically significant $(\mathrm{p}<0.05)$ in comparison to the $1^{\text {st }}$ and $3^{\text {rd }}$ collection

Urea levels showed a different behavior, there was a significant statistical increase $(p<0.05)$ in the $2^{\text {nd }}$ collection, demonstrating a change in renal filtration of this metabolic (Table 1). However, the $3^{\text {rd }}$ collection urea levels showed a decline, reaching almost the $1^{\text {st }}$ collection values.

Table 2 shows the lesions found in both kidneys of the 17 animals that were included in final analysis, summarized in values of percentage and absolute number of occurrence in animals.

TABLE 2 - Table shows the lesions found in both kidneys, summarized in values of percentage (absolute number) of occurrence in animals.

\begin{tabular}{lcc}
\hline Histopathologycal Changes & $\begin{array}{c}\text { Right Kidney } \\
\text { \% (n) }\end{array}$ & $\begin{array}{c}\text { Left Kidney } \\
\text { \% (n) }\end{array}$ \\
\hline $\begin{array}{l}\text { Cortical and Medullar } \\
\text { Congestion }\end{array}$ & $70.5(12)$ & $35.2(6)$ \\
Lymphocytic Infiltrate & $17.6(3)$ & $11.7(2)$ \\
Hydropic Degeneration Tubular & $5.8(1)$ & $33.2(6)$ \\
Cells & & \\
Bowman Area Increase & 0 & $11.7(2)$ \\
Glomerular Retraction & 0 & $11.7(2)$ \\
Repletion of Glomerular & $5.8(1)$ & $11.7(2)$ \\
Capillaries & & \\
Glomerulonephritis & $11.7(2)$ & $11.7(2)$ \\
Glomerular atrophy & 0 & $35.2(6)$ \\
Fibrosis & 0 & $11.7(2)$ \\
\hline
\end{tabular}

Histopathologycal changes in both kidneys: right and left. Total $\mathrm{n}=17$ animals

The histopathological analyzes showed that the injuries occurred in the renal tissue of the left kidney, which underwent the process of ischemia and reperfusion, and the right kidney, which did not undergo any intervention. These results observed in the righ kidney, are consistent with the process of reperfusion after ischemic induction since mediators that induce lesions in other tissues and organs not subjected to the ischemia are produced. 
Togoe EB et al.

The figures below show some of the histopathological lesions observed in the kidney tissue slides. In Figure 2 we can observe cortical and medullar congestion in the right and left kidney, in Figure 3 we can observe glomerular atrophy and hydropic degeneration in the left kidney and Figure 4 shows glomerulonephritis in the right kidney.
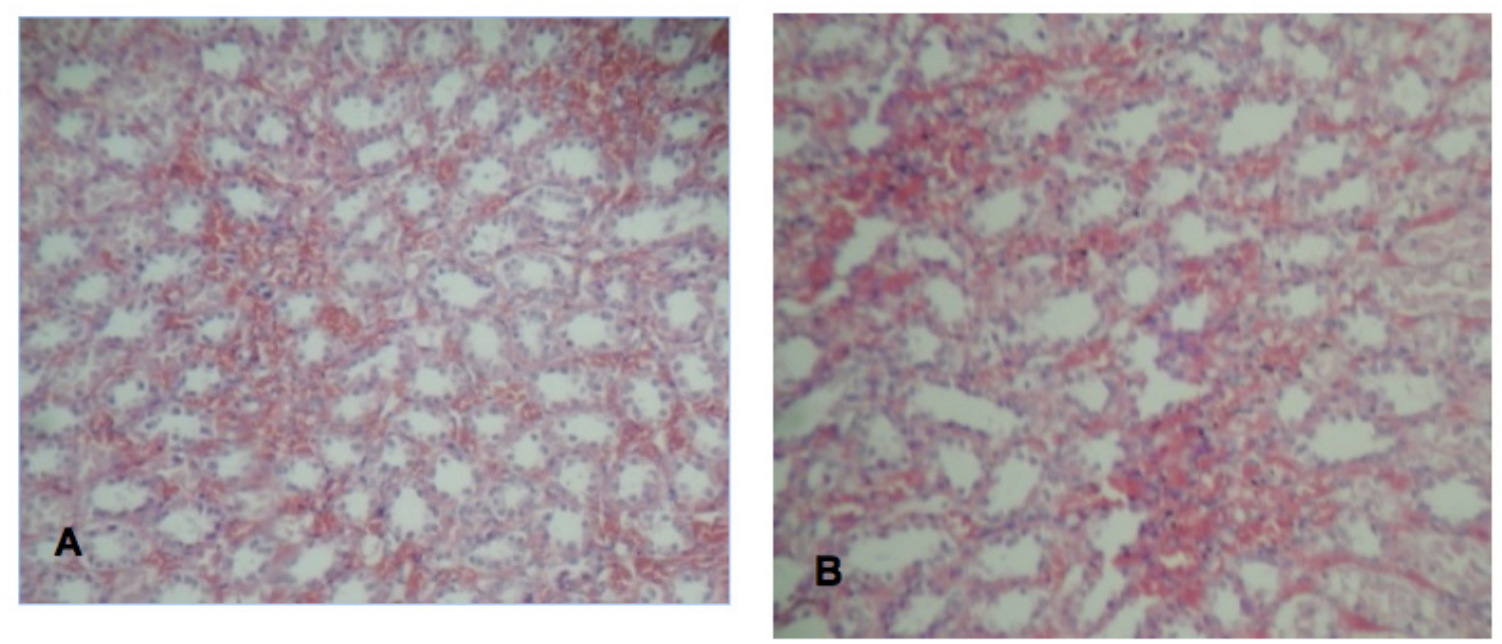

FIGURE 2 - Hystophatological slide photograph of left kidney (A) and right kidney (B) demonstrating cortical and medullar congestion (increase x10 HE).
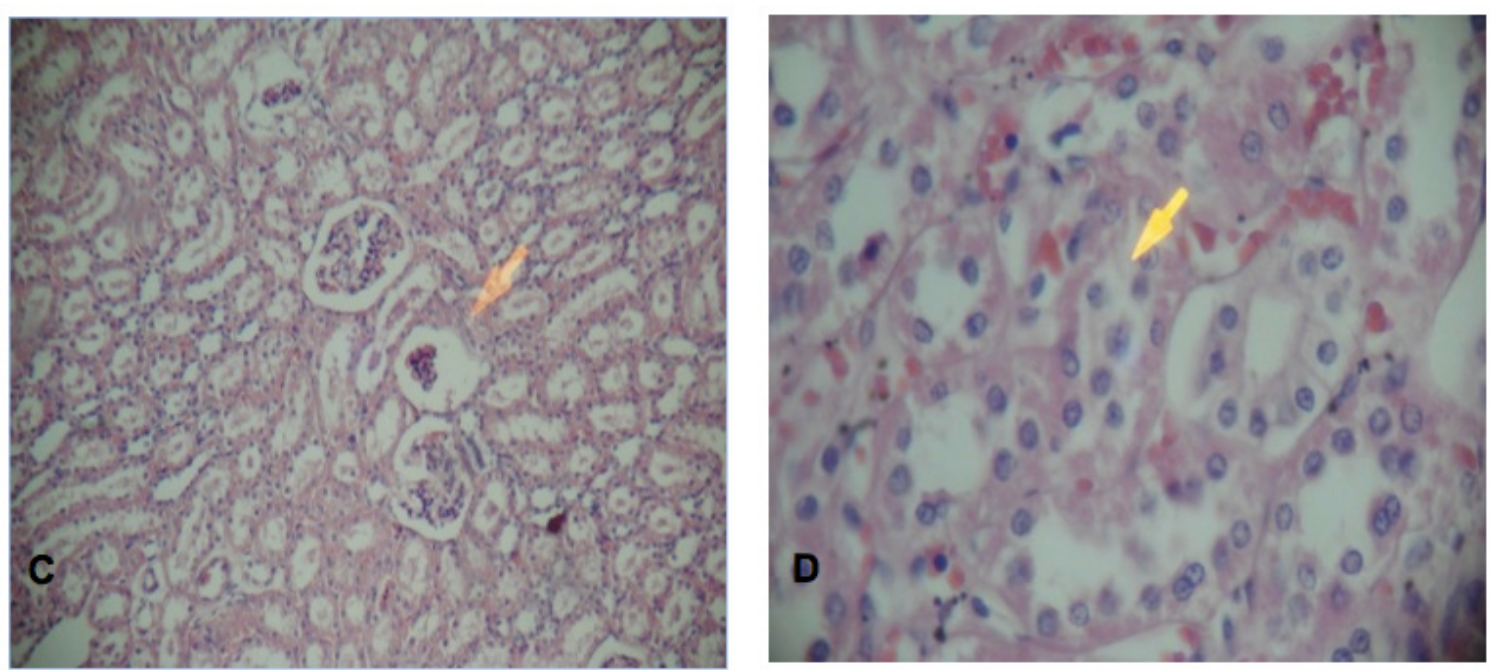

FIGURE 3 - Hystophatological slide photograph of left kidney showing glomerular atrophy (C) and hydropic degeneration of tubular cell (D) (increase $\mathrm{x} 40 \mathrm{HE}$ ).

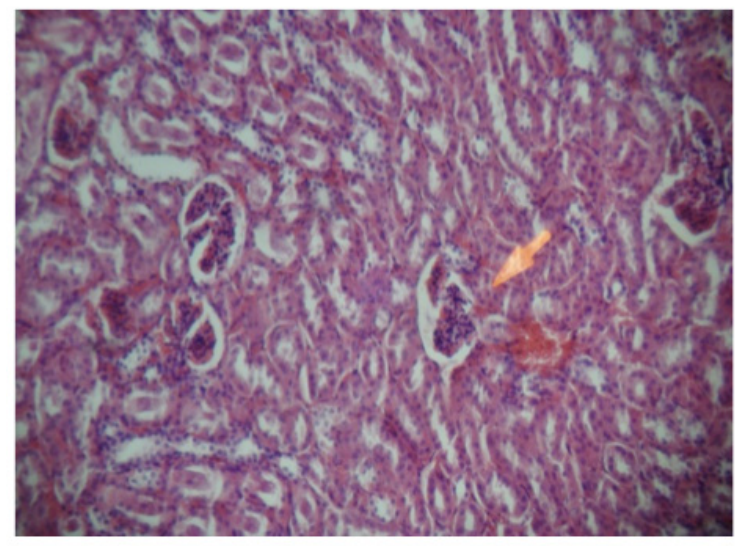

FIGURE 4 - Hystological slide photograph of the right kidneydemonstrating glomerulonephritis (increase x10 HE). 


\section{Discussion}

It was observed through this study that the unilateral technique of renal ischemia and reperfusion is feasible, and produces renal lesions compatible with CKD in White New Zealand rabbits.

Some studies conducted to establish an animal model of chronic kidney disease, used the two-surgical-times procedure, i.e., a first surgery is performed to establish the process of renal ischemia by clamping the unilateral renal pedicle and after a period that varied from four to 12 weeks, according to each protocol, a new surgical procedure is performed for the reduction of the renal mass to the contralateral organ that suffered the ischemic procedure ${ }^{5-7}$.

Santos et $a .^{5}$ examined the biochemical and morphological effects on the 60 remnant kidney's rats subjected to progressive ablation surgery of the renal mass equivalent to $1 / 2,2 / 3$ e 5/6, after eight weeks the animals were re-operated to remove the remaining kidney. This animal model showed considerable changes in the creatinine level, proteinuria and hypertrophy of the kidney, although the authors did not report the difficulties of this kind of animal model as well as the number of deaths.

Costa et $_{\text {al. }}{ }^{7}$ established a model of chronic renal failure in White New Zealand rabbits, using bipolar left nephrectomy and after four weeks, a right nephrectomy. The authors observed an increase progress in urea, creatinine, sodium and left kidney hypertrophy.

Knopka et al. ${ }^{6}$ evaluated a model of chronic renal ischemia in Wistar rats. The animals underwent a partial ligation of the left renal artery and after seven days a left nephrectomy. The authors observed a progressive reduction on weight, volume, renal cortical thickness, proximal tubular atrophy and infiltrate. The biochemical changes were not evaluated, however, according to the authors, the study provided a structural and morphological analysis of the animal model.

The available techniques in the literature induce changes consistent to the clinical course of CKD (increasing urea and creatinine levels, proteinuria and histopathological renal changes). However, they are difficult to be developed, since it involves twotime-surgery, which can lead to a reduction of the animal survival. Moreover, the choice of animal model, the Wistar rats, due to their limited size and low blood volemia, impair blood samples for biochemical analyzes and the reduction of animal survival in the post-surgery $^{5-7}$. Thus, in this experiment we chose to use the White New Zealand rabbits and one surgical time procedure, through the exclusive unilateral technique of renal ischemia and reperfusion.

The choice of the White New Zealand Rabbit for this experiment was due to their better survival after CKD induction, with the possibility of providing a therapeutic window of 12 weeks agreeing with Costa et al. ${ }^{7}$. When considering the life expectancy of a rabbit, around seven to eight years, approximately ten times smaller than human beings, we can assume that the observation interval of 12 weeks is equivalent to two and a half years on anima móbile, which features enough time for $\mathrm{CKD}$ installation. Moreover, they present a higher volemia, compared to the rats, facilitating the samples collections for biochemical analysis. In the present experiment, consecutive blood samples for biochemical analysis were taken, without compromising the lives of animals that remained viable around 11 weeks until euthanasia.

The exclusive unilateral technique of renal ischemia and reperfusion led to a continuous increasing in creatinine levels after the CKD induction. Renal clearance of creatinine has a long history as a marker of GFR. Spanaus et al. ${ }^{12}$ presented a study that strengthens the use of creatinine as a reliable marker for CKD. These authors compared the performance of two low molecular weight markers, cystatin $\mathrm{C}$ and $\beta$-trace protein (BTP), with creatinine serum for the diagnosis, staging and prediction of progression in a cohort of 227 patients with primary non-diabetic $\mathrm{CKD}$ and concluded that the three biomarkers are equivalent in terms of diagnostic performance - even in discrete degrees of deterioration of renal function - and in terms of predicting risk for progression. These authors stated that any serum biomarker of GFR should obey the laws of physics: with declining GFR, serum concentration should increase. The fact of the relationship between serum concentration and GFR be a reciprocal function, explains how relatively small changes in concentration, that occur in the early stages of decline in kidney function are followed by accelerated increases. Thus, when analyzing the data obtained in blood samples in this study after 11 weeks of renal ischemia and reperfusion procedures, serum concentrations increased consistent to the decreased of glomerular filtration.

During the surgical procedure, to generate ischemia and reperfusion, the left renal pedicle was clamped for 20 minutes. It was believed that in comparison to the heart and brain, the kidneys could recover completely from an ischemic intervention. However, this has been occurring with less frequency, recognizing that ischemia/reperfusion (more than 20 minutes) can lead to $\mathrm{CKD}^{13}$. Thompson et $a l .{ }^{14}$ studying the risk related to the time of renal ischemia in clinical impact, suggests that the vascular clamping of the renal pedicle is associated with the increased risk of chronic deterioration of renal function. His study demonstrated that ischemia for more than 20 minutes is associated with the development of $\mathrm{CKD}^{14}$. In our experiment a 20 minuterenal ischemia was sufficient to generate lesions proved by histopathological analyzes. 
Renal ischemia/reperfusion injury is the result of a deficiency of oxygen and nutrient delivery to the removal of wasting through tubular cells. There is a mismatch between the demand and supply of $\mathrm{O}_{2}$, with the accumulation of substances, metabolism products. As a result, of an imbalance, cells of the renal tubular epithelium suffer severe damage, such as cell death by apoptosis and necrosis, creating disturbances in the water and electrolyte homeostasis and consequent reduction in the excretion of waste products from metabolism ${ }^{8}$.

One reason for the renal sensitivity ischemia is that the renal microvasculature is highly complex, and must meet a high demand for energy. This delicate balance between supply and demand for $\mathrm{O}_{2}$ is easily disturbed in cases of ischemia ${ }^{15}$. A study has shown that during ischemia/reperfusion blood flow to the renal medulla is reduced disproportionally to the reduction of renal perfusion. The vascular endothelium is injured and small renal arterioles undergo vasoconstriction in response to high tissue levels of chemical mediators of inflammation (endothelin-1, angiotensin II, thromboxane A2, and leukotriene) and stimulation of the peripheral nervous system. In addition, there is a decrease of the vasodilatation of arterioles due the decline of nitric oxide and other vasodilators, associated to the increase of vasoactive cytokines (TNF- $\alpha$, IL, endothelin), generating as a result, activation and adhesion of leukocytes to the endothelium. The edema formation further contributes to arteriolar vasoconstriction, resulting in interference on the blood flow to the proximal tubule and loop of henle, which are usually hypoxic due to exchange properties with straight renal vessels ${ }^{8}$.

Clinically all these changes reflect an increased injury during the reperfusion phase, when the $\mathrm{O}_{2}$ consumption increases with the demand of tubular transport. Thus, the initial injury leads to a cascade of events that culminate in vascular injury, vascular congestion and reduced ability to neutralize the toxic radicals, a fact that undermines the renal filtration and leads to a progressive increasing levels of urea and creatinine ${ }^{16}$. Corroborating the literature, this experiment demonstrated the loss of renal function through the progressive increase in creatinine levels, caused by unilateral ischemic process.

The consequence of an ischemic process of renal injury is an important cause of CKD in humans. In this sense, Arterial Hypertension (AH) plays an important role in renal ischemia because both accelerated and chronic forms may provide a framework for kidney injury of microvascular nature, characterized by myointimal proliferation and fibroid necrosis. Hyaline arteriosclerosis that installs due to $\mathrm{AH}$ generates the process with consequent ischemic injury of renal microvasculature, featuring hypertensive nephrosclerosis. Considering the high prevalence of hypertension in the population, a significant number of patients with renal dysfunction can be predicted and identified in our environment as the second leading cause, after diabetic nephropathy in patients starting dialysis each year, according to data of the Health Department of Brazil ${ }^{17,18}$.

This exclusive unilateral technique of renal ischemia and reperfusion is quite useful for the $\mathrm{CKD}$ induction process, as renal ischemia is related to diseases so prevalent in the community, such as Arterial hypertension and Diabetes Mellitus ${ }^{19}$.

\section{Conclusion}

This experiment using an exclusive unilateral technique of renal ischemia and reperfusion, without a second-time surgery for nephrectomy, in White New Zealand rabbits, showed effectiveness in getting an animal model of Chronic Kidney Disease.

\section{References}

1. Sesso RC, Lopes AA, Thomé FS, Lugon JR, Watanabe Y, Santos DR. Chronic dialysis in Brazil: report of the Brazilian dialysis census, 2011. J Bras Nefrol. 2012 Jul-Sep;34(3):272-7. PIMD: 23099833.

2. Sesso RC, Lopes AA, Thome FS, Lugon JR, Watanabe Y, Santos DR. Report of the brazilian chronic dialysis census 2012. J Bras Nefrol. 2014 Mar;36(1):48-53. PIMD: 24676614.

3. Martínez-Ramírez HR, Cortés-Sanabria L, Rojas-Campos E, Hernández-Herrera A, Cueto-Manzano AM. Multidisciplinary strategies in the management of early chronic kidney disease. Arch Med Res. 2013 Nov;44(8):611-5. doi: 10.1016/j. arcmed.2013.10.013.

4. Nitta K, Okada K, Yanai M, Takahashi S. Aging and chronic kidney disease. Kidney Blood Press Res. 2013;38(1):109-20. doi: 10.1159/000355760.

5. Santos LS, Chin EW, Ioshii SO, Tambara Filho R. Surgical reduction of the renal mass in rats. Morphologic and functional analysis on the remnant kidney. Acta Cir Bras. 2006 Jul-Aug;21 (4):252-7. PMID: 16862347.

6. Konopka CL, Jurach A, Wender OC. Experimental model for the study of chronic renal ischemia in rats: morphologic, histological and ultra-structural analysis. Acta Cir Bras. 2004 Jan-Feb;22 (1):1221. PMID: 17293944.

7. Costa AF, Pereira Lde P, Ferreira ML, Silva PC, Chagar VL, Schanaider A. Surgical model of chronic renal failure: study in rabbits. Rev Col Bras Cir. 2009 Feb;36 (1):78-84. PMDI: 20076872.

8. Bonventre JV, Yang L.Bonventre JV, Yang L. Cellular pathophysiology of ischemic acute kidney injury. J Clin Invest. 2011 Nov;121(11):4210-21. doi: 10.1172/JCI45161. Epub 2011 Nov 1.

9. Fougeray S, Bouvier N, Beaune P, Legendre C, Anglicheau D, Thervet E, Pallet N. Metabolic stress promotes renal tubular inflamacion by triggering the unfoldead protein respose. Cell Death Dis. 2011 Apr 14;2:e143. doi: 10.1038/cddis.2011.26.

10. Bastos MG, Bregman R, Kirsztajn GM. Chronic kidney diseases: common and harmful, but also preventable and treatable. Rev Assoc Med Bras. 2010 Mar-Apr;56(2):248-53. PMID: 20499004. 
11. Peres LA, Matsuo T, Delfino VD, Peres CP, Almeida Netto JH, Ann HK, Camargo MT, Rohde NR, Uscocovich VF.Increase in prevalence of diabetes Mellitus as a cause of dialytic end-stage renal disease - Analysis of 20 Years in the West Region of Paraná. Arq Bras Endocrinol Metabol. 2007 Feb;51(1):111-5. PMID: 17435864.

12. Spanaus KS, Kollerits B, Ritz E, Hersberger M, Kronenberg F, von Eckardstein A; Mild and Moderate Kidney Disease (MMKD) Study Group. Serum creatinine, cystatin $\mathrm{C}$, and beta-trace protein in diagnostic staging and predicting progression of primary nondiabetic chronic kidney disease. Clin Chem. 2010 May;56 (5):740-9. doi: 10.1373/clinchem.2009.138826.

13. Ishani A, Xue JL, Himmelfarb J, Eggers PW, Kimmel PL, Molitoris BA, Collins AJ. Acute kidney injury increases risk of ESRD among elderly. J Am Soc Nephrol. 2009 Jan;20(1):223-8. doi: 10.1681/ ASN.2007080837.

14. H, Frank I, Lohse CM, Saad IR, Fergany A, Zincke H, Leibovich BC, Blute ML, Novick AC. The impact of ischemia time during open nephron sparing surgery on solitary Kidneys: a multi-Institutional study. J Urol. 2007 Feb;177(2):471-6. PMID: 17222613.

15. Sutton TA. Alteration of microvascular permeability in acute kidney Injury. Microvasc Res. 2009 Jan;77(1):4-7. doi: 10.1016/j. mvr.2008.09.004.

16. Munshi R, Hsu C, Himmelfarb J. Advances in understanding ischemic acute kidney injury. BMC Med. 2011 Feb 2;9:11. doi 10.1186/1741-7015-9-11.

17. Levin A, Hemmelgarn B, Culleton B, Tobe S, McFarlane P, Ruzicka M, Burns K, Manns B, White C, Madore F, Moist L, Klarenbach S, Barrett B, Foley R, Jindal K, Senior P, Pannu N, Shurraw S, Akbari A, Cohn A, Reslerova M, Deved V, Mendelssohn D, Nesrallah G, Kappel J, Tonelli M; Canadian Society of Nephrology. Guidelines for the management of chronic kidney disease. CMAJ. 2008 Nov 18;179(11):1154-62. doi: 10.1503/cmaj.080351.

18. Condé SA, Fernandes N, Santos FR, Chouab A, Mota MM, Bastos MG. Cognitive decline, depression and quality of life in patients at different stages of chronic kidney disease. J Bras Nefrol. 2010 JulSep;32(3):242-8. PMID: 21108636.

19. Singh P, Rifkin DE, Blantz RC. Chronic kidney disease: an inherent risk factor for acute kidney injury? Clin J Am Soc Nephrol. 2010 Sep;5(9):1690-5. doi: 10.2215/CJN.00830110.

\section{Acknowledgements}

To Profa. Bruna Oliveira Parente and Profa. Vanessa Menegatti Marcondes for biochemical analysis, veterinary Camila Aparecida Nascimento for histopathological preparations, Profa. Fernanda Zauith for allows the locations for this research and the Veterinary Hospital Unit staff for contributes to care of the animals.

\section{Correspondence:}

Eliane Barbosa Togoe

Rua Ciro Melo, 3273

79805-031 Dourados - MS Brasil

elianetogoe@hotmail.com

Received: May 19, 2014

Review: July 21, 2014

Accepted: Aug 22, 2014

Conflict of interest: none

Financial source: none

${ }^{1}$ Research performed at Veterinary Hospital Unit, University Center of Grande Dourados (UNIGRAN), Dourados-MS, Brazil. Part of Master degree and $\mathrm{PhD}$ thesis, Postgraduate Program in Health and Development, Federal University of Mato Grosso do Sul (UFMS), Campo Grande-MS, Brazil. Tutors: Prof. Iandara Schettert Silva (Master degree thesis) and Albert Schiaveto Souza (PhD degree thesis). 